\title{
Study on the chessboard recognition and positioning method of chess game system
}

\author{
Huibai Wang, Xiao Liu, Ming Shi \\ College of Information Engineering, North China University of Technology, Beijing, China \\ E-mail: hb2008Ix@ncut.edu.cn
}

\begin{abstract}
Keywords:augmented reality; SIFT feature matching; k-means clustering; chessboard recognition; checkerboard positioning
\end{abstract}

\begin{abstract}
With the wide application of augmented reality technology, designing a remote chess game based on augmented reality technology has the research significance of potential. The system can make two people both living in different places to play chess as they are playing face to face and can communicate with each other. This paper presents a new methods for identifying the chess board areas in the scene which based on SIFT feature matching and K-means clustering. This paper presents a method of automatically obtaining segmentation threshold based on the parameters of the linear distance difference which can used to exclude the extra line detected and ultimately determine the linear model of $9 * 10$. The experiment proved that the method proposed in this paper can effectively remove the restricted conditions that the camera can only shoot board area and the camera must be taken perpendicular to the board and the method can coordinate position of accurate positioning checkerboard. This method can be reference for similar problems.
\end{abstract}

\section{Introduction}

In recent years, with the development of image processing technology and augmented reality technology matures, entertainment has changed a lot so designing a remote chess game based on augmented reality technology has the research significance of potential. Firstly, the camera shooting each other chess scene, compress the data of the other scene, transmit the data to the local computer Real time. Secondly, acquire the information of the pieces of positions and motions, and the images of hand motions in the local scene. Finally, the virtual chess pieces which designed according to the true pieces will be superimposed to remote image according to the position information, and at the same time the local hand motion will be synthesis to remote image. This eventually achieve two people can share a chessboard for chess as well they can see each other's manners, hear voices just as they were in the same scene, enhancing the feeling of immersion during recreation. There are lots of difficulties and key technology in the design and implementation of the system. In the system, how to detect the chess board efficiently and accurately and how to locate the chessboard coordinates in the regions scene are the important basis for the later work. There are many scholars at home and abroad in-depth study related to this technology. Among them, Dr. Zhang Zhiwei of University of Science \& Technology China proposed a method of segmentation which according to the obvious difference of saturation between the chessboard and outsize board in the S component of HIS image. Dr Du Junli of the Huazhong University of Science and Technology put forward chess binary images method based on pixel difference threshold to detect the chessboard. Master Zhai Naiqiang puts forward a method of China chessboard recognition based on collaborative work of recognition of color and character. Through the study and research, found there are very strong restrictions on camera in checkerboard test stage. Generally the camera id restricted to place above the board area, and the acquisition obtained image is mainly the chess board regional image. The remote chess system base on augmented reality is to enable both sides to play chess is more able to feel the sense of immersion mainly, so the scene range should not only the chessboard area and the camera should not just above the chessboard. Combined with many research techniques, this article will introduce how to detect the checkerboard areas and how to carry out the chessboard coordinate in complex scenes. 


\section{SIFT feature extraction}

SIFT is a local feature detection algorithm, and its essence is to find the feature points in different scale space, these feature points with scale invariance in the property, position, rotation. The algorithm can be divided into the following steps: generating the scale space, to obtain extreme values of the scale space, confirmation the position of feature points, determining the characteristic direction, generating feature point descriptor.

The 2D gauss function is defined as follows:

$$
\mathrm{G} x, \mathrm{y}, \sigma)=\frac{1}{2 \pi \sigma^{2}} e^{-\left(x^{2}+y^{2}\right) / 2 \sigma^{2}}
$$

In this equation, $\sigma$ represents the variance of Gauss normal distribution, is the scale coordinate. In the equal $I(x, y)$ represents the input images, which can be obtained by image and the Gauss kernel convolution by different scale space representations:

$$
L(x, y, z)=G(x, y, \sigma) * I(x, y)
$$

Scale space presented by Gauss Pyramid, and Gauss differential image can be obtained by the adjacent the upper and the lower layers of the Gauss Pyramid.

$$
\begin{aligned}
& \mathrm{D}(\mathrm{x}, \mathrm{y}, \sigma) \\
& =(\mathrm{G}(\mathrm{x}, \mathrm{y}, \mathrm{k} \sigma)-\mathrm{G}(\mathrm{x}, \mathrm{y}, \sigma)) * \mathrm{I}(\mathrm{x}, \mathrm{y}) \\
& =\mathrm{L}(\mathrm{x}, \mathrm{y}, \mathrm{k} \sigma)-\mathrm{L}(\mathrm{x}, \mathrm{y}, \sigma)
\end{aligned}
$$

The constant coefficient $\mathrm{k}$ is used to distinguish between adjacent scales. The feature points in the image can be obtained by the local extreme points in the Gauss differential image. The main direction and mode value of gradient of the feature point can be got from the (4) and (5):

$$
\begin{aligned}
& m(x, y) \\
& =\sqrt{(L(x+1, y)-L(x-1, y))^{2}+(L(x, y+1)-L(x, y-1))^{2}} \\
& \theta(x, y)=\tan ^{-1}\left(\frac{(L(x, y+1)-L(x, y-1)}{L(x+1, y)-L(x-1, y)}\right)
\end{aligned}
$$

Finally, a descriptor should be established for each feature point which represent the feature point and ensure it does not change with the variety of the changes. The descriptor contains the key point and the surrounding pixels contribute to the key point. According to dividing the descriptors into blocks and calculating the gradient histogram in the blocks generate the unique vector. The vector is the abstract of the area of the image information which is unique. A SIFT feature descriptor is generated.

\section{K-means}

\section{A. Introduction K-means Algorithm}

Clustering analysis as a non supervised learning method is an important research direction in the field of machine learning. At the same time, clustering techniques is also important analytical tools and methods of data processing of data mining. Macqueen was first proposed K-means clustering algorithm in 1967 (K-means algorithm). K-means clustering algorithm is a basic algorithm which often uses the squared error criterion as the clustering criterion function. So far, many have opted for the classic task clustering algorithm.

\section{B. K-means Algorithm Steps}

The basic idea K- means clustering algorithm is that chose $\mathrm{k}$ points as the center of clustering, using the nearest principle, set the rest of the data set object assigned to the $\mathrm{K}$ classes. Clustering results expressed by the $\mathrm{K}$ clustering center. Algorithm uses iterative updating method, each iteration process is performed to the objective function value decreasing direction by comparing to the given objective cluster function. The points will be set into $\mathrm{k}$ classes according to the $\mathrm{k}$ 
reference points and the geometric center of each class will be as the reference points at the next iteration. Iteration makes the selected reference point closer to the real geometric center of the class and makes the similarity within the class of objects greatest and similarity between the classes of objects smallest.

Main steps:

Choose $k$ objects from the entire sample randomly as initial cluster centers: $m_{i}(\mathrm{i}=1,2,3, \ldots \mathrm{k})$; $d\left(p, m_{i}\right)$

Using equation (6) calculate the distances from each data $\mathrm{p}$ to the cluster centers;

Find the minimum $d\left(p, m_{i}\right)$ of each object, classified the $\mathrm{p}$ into a same cluster;

After all the objects traversed, using (7) to recalculate the value of $m_{i}$, as the new cluster center cluster;

Re-dividing the whole data set objects to the most similar clusters cluster, this procedure is repeated until a minimum squared error criterion;

$$
\begin{gathered}
d(i, j)= \\
\sqrt{\left(x_{i 1}-x_{j 1}\right)^{2}+\left(x_{i 2}-x_{j 2}\right)^{2}+\ldots+\left(x_{i n}-x_{j n}\right)^{2}} \\
i=\left(x_{i 1}, x_{i 2}, \ldots, x_{i n}\right), j=\left(x_{i 1}, x_{i 2}, \ldots, x_{i n}\right) \text { are two n-dimensional data objects. } \\
m_{k}=\frac{\sum_{i=1}^{N} x_{i}}{N}
\end{gathered}
$$

\section{Detection of remote chess game board system}

\section{Checkerboard Detection Based on Combination of SIFT Feature Matching and K-means Clustering}

Because of the range of scenarios involving relatively large, not just the area of the board, when the board area is detected the impact of other regions should be excluded. Because chess board itself has some unique features, such as color of the basic unity of the board area (Figure 1), so the data of the image could be got into different types of data according to the k-means algorithm, which will be able to board area and the non checkerboard region separated.

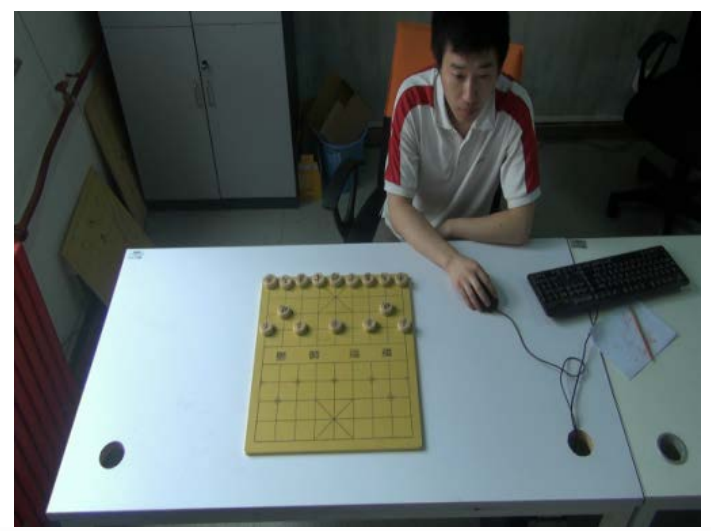

Figure 1 Scene Image Containing Checkerboard

In the use of K-means algorithm to divide the board area, may be because of the color of the pieces or the color of character of pieces and board area is inconsistent, leading to the phenomena that there will be some empty internal during the regional division(Figure 3). The internal cavity filled, a complete extraction checkerboard region can be obtained. The empty chessboard area obtained could be filled according to certain morphological processing. The internal board area empty will be filled by expansion processing but this will have magnified the chessboard area, therefore corresponding corrosion processing is needed to resize the enlarged board to original size (Figure 8). 
Through the K-means algorithm and morphology processing we can segmentation the chessboard from the entire scene very well, but we are not able to know which part is the board regional. Due to the presence of unique feature point in the board, it is possible to obtain the feature points and at the same time match the feature points in the scene according to the SIFT and finally obtain the point pairs. And calculated on the number of point pairs occupied in various parts. Because the feature point is extracted from the board, so the aggregation area of the feature point of the scene is the chessboard detected.

\section{A. Process Design of Checkerboard Detecting}

Board detection algorithm proposed in this paper is the combination of k-means clustering and SIFT feature matching to detect complex scene checkerboard area.

Extract feature points checkerboard area based on SIFT algorithm from the image which has a separate area of the chessboard.

To obtain an image of the scene through the camera containing the board area, and extract feature points based on SIFT algorithm in the whole scene.

Matching feature points extracted from board region and feature points obtained from the entire region and the matching points will be stored.

RGB images contain scenes checkerboard region will get converted to HSV image in order to minimize the impact of light on the data.

HSV image data based on k-means algorithm gets into different categories, and morphological processing, so that the board region can be separated from other regions.

Calculated the number of the saved matching point pairs occupied in various parts according to k-means classification.

The aggregation area of the feature points of the scene is the chessboard area.

\section{Experimental Analysis and Results}

In this study, environmental parameters as the following:

The experimental environment parameters are as follows: computer is Dell Precision T5600, CPU is E5-2603 0@1.80GHz (2 processors), memory is $8.00 \mathrm{~GB}$, and the operating system is Window 7 Professional Edition (64-bit operating systems), simulation platform for Visual C ++ 6.0.

\section{A. Classify the Image In the Scene Use of k-means}

First, convert the image of the scene into HSV image data in order to reduce the impact of light on the classification of image data. In this study, experimental data are divided into four categories, which mean scene data will be divided into four categories, as Fig. 2 and Fig. 3 shown.

We can get four binary images of different categories according to the classification of the image data, as Fig. 4 to Fig.7 shown.

The empty emerged on the board and border can be Morphological filled. That is the image will be conducted an inflate process firstly and then resized the image to regional size by etching treatment. The empty in the board area will be filled as Fig. 8 to Fig.11 shown.

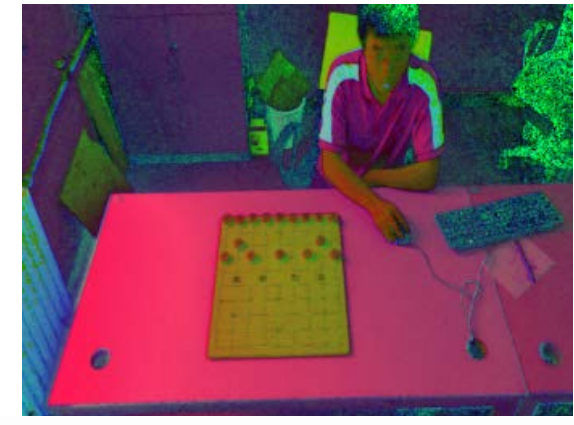

Figure 2 HSV image after conversion

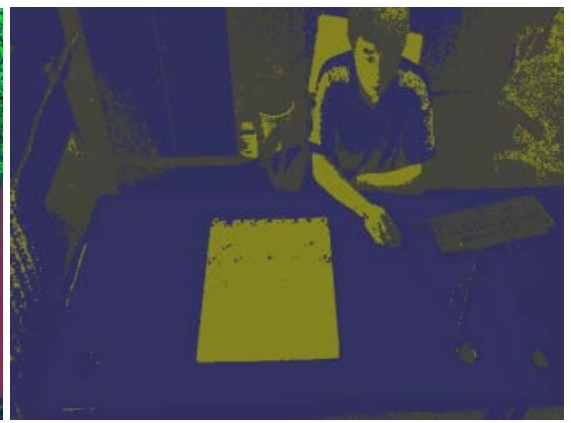

Figure 3 Image after classification 


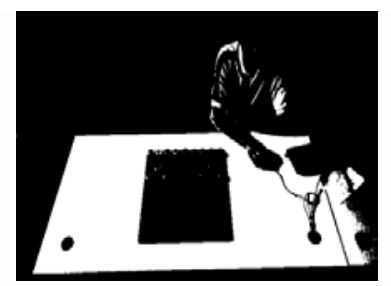

Figure 4 Binary image of category 1

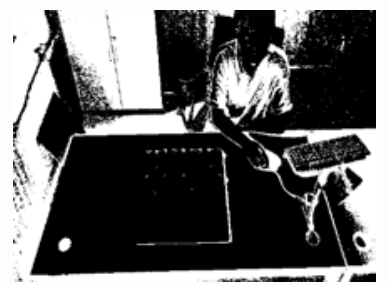

Figure 6 Binary image of category 3

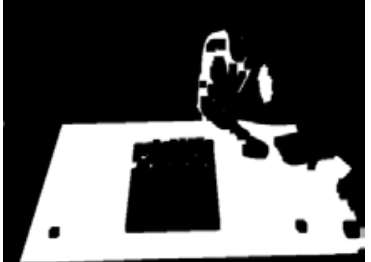

Figure 8 Processed of Fig. 4

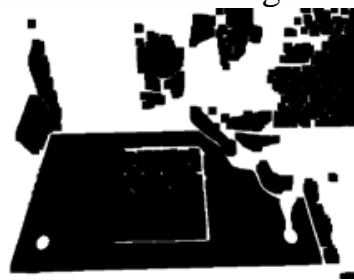

Figure 10 Processed of Fig. 6

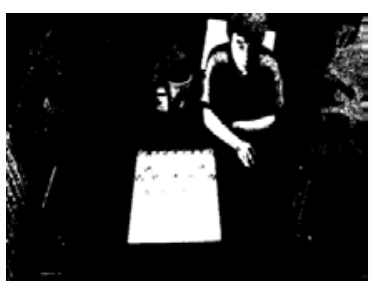

Figure 5 Binary image of category 2

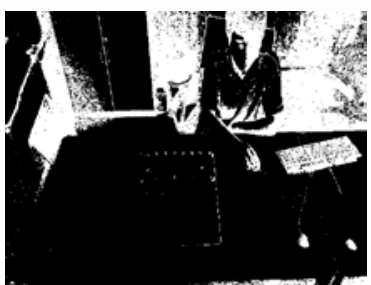

Figure 7 Binary image of category 4

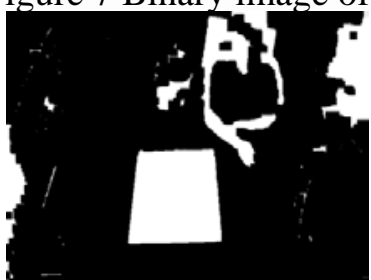

Figure 9 Processed of Fig. 5

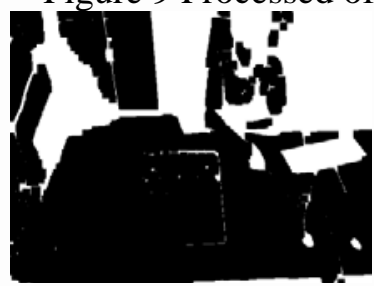

Figure11 Processed of Fig. 7

\section{B. Obtain Matching Points By SIFT Feature Matching Algorithm}

Because our purpose is to identify checkerboard area in the scene, we must first capture the checkerboard area image and get feature points in the checkerboard area obtained. As the Fig.12 show, the extracted image size is $240 * 240$ and 40 feature points were extracted in the board area.
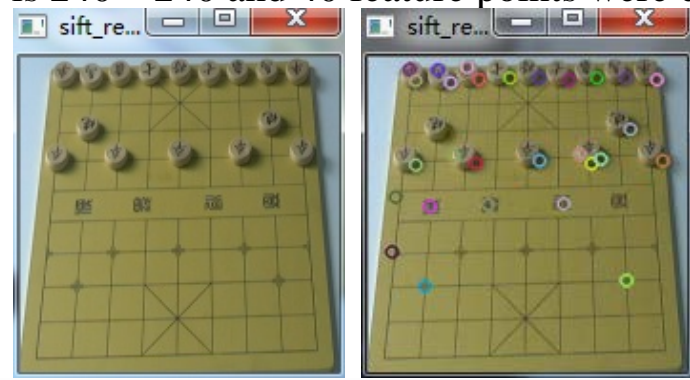

Figure12 Chessboard image Figure13 Feature points in checkerboard area
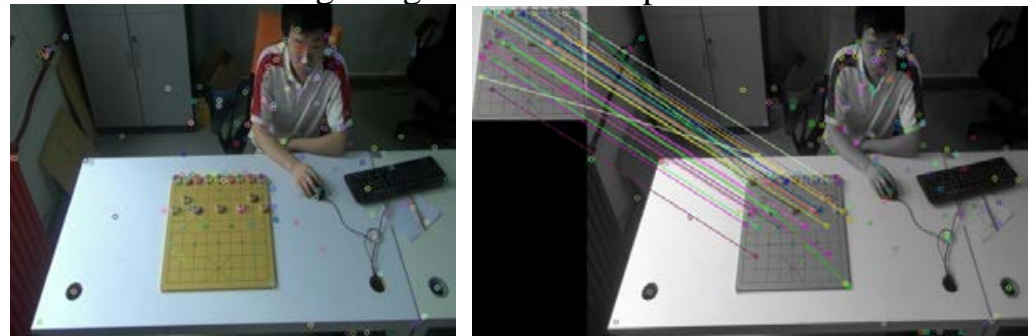

Figure14 extracting feature points in the scene Figure15 Matching the feature points in two sets

Acquiring an image of the whole scene, and gets its feature points. The size of image of scene is $640 * 480$ and 211 feature points were extracted in the image, as shown in Fig.14.

Initial matching is matching feature points acquired from the checkerboard area and scene area according to simple pattern matching algorithm and approximate matching point pairs will be got. 
The result is shown in Fig.15.

Through the experimental image we can know, there will be some error matching points in the feature point matching.
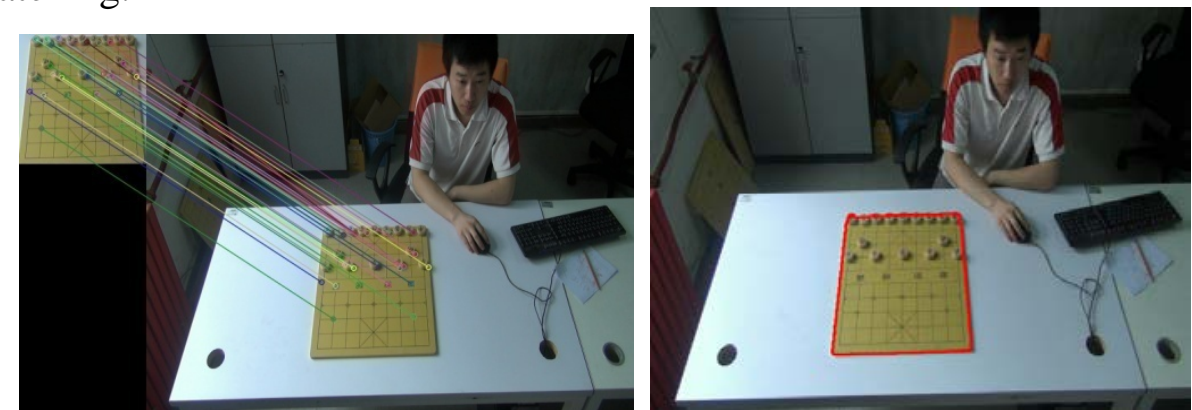

Figure16 Eliminating the Part of Wrong Matching Points Figure 17 Detecting Chessboard Area

In this experiment, using RANSAC algorithm to eliminate part of the wrong matching points. Finally get the thirty-four matching points, and store the above thirty-four matching points in the scene coordinates.

\section{Counting Matching Points and Obtain the Chessboard Area}

Counting the number of acquired matching points is occupied in four kinds of images, to determine the location of chessboard area. In this experiment, the chessboard area location is the second kinds of images. And the thirty-four matching points just in the location of chessboard area. Thus we can obtain the chessboard area, and mark the acquired chessboard area. The result will be show As the Fig.17.

\section{Conclusions}

This paper presents a new methods for identifying the chessboard areas in the scene which based on SIFT feature matching and K-means clustering. It can effectively solve the problem ofcamera shooting position. Makes the scene not only display chessboard area, greatly expanded the scope of scene, and accurately extracted on the chessboard area in such complex scenario. It plays an important role in the later design of remote chess system. Some design methods proposed in this paper, have certain enlightenment function for the same type of board games.

\section{References}

[1] Du Junli,Zhang jingfei, Huang Xinhan, "Based on Visual Recognition Chessboard”, Computer Engineering and Application,Feb 2007,pp.220-222.

[2] Zhai naiqiang,’'Improved Chinese chessboard recognition methocd” , Journal of Computre Application,Vol.30 No.4,Apr.2010,pp.980-981.

[3] Bi jintao,"Reaseach on Chinese-chess Playing Robot Control System”, Harbin Polytechnic University, Mar,2009.

[4] Zhang zhiwei, Kong fanrang, Zhao jiwen, He qingbo, Wu zengrong,”Image Processing and Recognition in the Vision of Chinese Chess Playing Robot", Computer Applications and Software,Voi.25 No.2, Feb,2008, pp.215-217.

[5] Du Junli, Huang Xinhan,”Design of Chinese Chess Robot Vision System”, Application of Electronic Technique, Sept, 2007,pp.133-136.

[6] Fu qiang, Chen huanwen,"Research on Methods of Self-Teaching of Chinese Chess Game”, Computer Tecnology and Development, Vol.17, No.12, Dec 2007,pp.76-79.

[7] Xu Xiaogang, Yu Jinhui, Ma Lizhuang, Extraction of Complex Object Contour, Journal of Image and Graphics, Oct,2001, pp.455-459.

[8] Nitin Aggarwal, William Clem Karl, Line Detection in Images Through Regularized Hough Transform, IEEE Signal Processing Society, 2006,pp.582-591. 